\title{
A NEGLECTED CASE OF PRIMARY INFERTILITY - A CASE REPORT OF DISSEMINATED TUBERCULOSIS
}

Nidhi Bansal, Hiremath P.B, Reshma Hiremath, Meenal C. Arun Kumar S.P., Madhumitha S, Kalyani Kalli.
1. Assistant Professor. Department of Obstetrics \& Gynaecology, SVMCH \& RC, Ariyur, Pondicherry.
2. Associate Professor. Department of Obstetrics \& Gynaecology, SVMCH \& RC, Ariyur, Pondicherry.
3. IMO. Mapusa, Goa.
4. Professor. Department of Obstetrics \& Gynaecology, SVMCH \& RC, Ariyur, Pondicherry.
5. Professor. Department of Pathology, SVMCH \& RC, Ariyur, Pondicherry.
6. Junior Resident. Department of Obstetrics \& Gynaecology, SVMCH \& RC, Ariyur, Pondicherry.
7. CRRI, Department of Obstetrics \& Gynaecology, SVMCH \& RC, Ariyur, Pondicherry.

\section{CORRESPONDING AUTHOR:}

Dr. Hiremath P.B,

Associate Professor of OBG,

SVMCH \& RC, Ariyur-605102.

E-mail: hiremath0312@gmail.com

KEYWORDS: Genital tuberculosis, Infertility, Bicornuate uterus, Diagnostic laparoscopy, invitro fertilization

ABSTRACT: Tuberculosis (TB) is a major health problem in developing countries including India and genital tuberculosis (GTB) is responsible for infertility in a significant proportion of these women. Inspite of recent advances in medicine and availability of sophisticated investigative modalities, definitive diagnosis of GTB is elusive in absence of high clinical suspicion. Majority of women with genital tuberculosis present with infertility. Oligomenorrhea or amenorrhea is more common with other vague symptoms. Laparoscopy is now a well recognized procedure in the diagnostic work up of patients with infertility. In a clinically suspicious case, endometrial biopsy (EB) should be followed by laparoscopy. The treatment of GTB should begin with anti tuberculous therapy. Pregnancy rates can now be significantly improved with In vitro fertilization (IVF). The fact remains that fertility rates are not optimistic in genital TB patients even after treatment.

INTRODUCTION: Tuberculosis (TB) is a major health problem in developing countries including India and genital tuberculosis (GTB) is responsible for infertility in a significant proportion of these women ${ }^{1}$.It is difficult to assess the actual incidence of genital tuberculosis accurately, as the disease is discovered incidentally in many patients and majority of them are asymptomatic ${ }^{2}$. It is estimated that $5-10 \%$ of infertile women worldwide have GTB, although this varies from less than $1 \%$ in the United States to nearly $18 \%$ in India ${ }^{2}$. When TB affects genital organs of young females, it produces devastating effects by causing irreversible damage to the fallopian tube resulting in infertility which is difficult to cure both by medical and surgical methods. 3,4

CASE REPORT: A 28 year old female, a case of primary infertility for 8 years, with irregular cycles, 2/15-20 days with scanty flow came to our gynaecology out-patient department. On history, there were no symptoms suggestive of any chronic systemic diseases related to infertility. 
No significant family history. She gave a history of multiple gynecological evaluations for infertility over the past 7 years. Semen analysis was normal. Hormonal assay which was done one year back was normal ( Thyroid function test, Serum prolactin, Serum FSH, LH, Serum Estriol, progesterone etc).USG showed uterus didelphys with an hypoechoic cyst with internal echoes measuring $8.2 \times 8 \times 9.2 \mathrm{~cm}$ seen in the adnexa ( site could not be identified due to large size of the cyst) , left adnexa appears normal. MRI done outside showed a hypoplastic uterus with bicornuate unicollis type of mullerian duct anomaly with endometriotic change in left uterine horn with small collection, both the ovaries appear unremarkable. HSG showed uterus of bicornuate, probably unicollis variety, irregularities and crenations are noted in the uterine horn silhouettes - probably endometritis. Both the tubes are visualised almost throughout their extent, no peritoneal spillage of contrast is visualized. Soft tissue circumscribed calcifications are noted across the left hemipelvis-? Adnexal. Repeat USG at our centre showed no evidence of adnexal cyst but identified multiple calcifications in the pelvis. $\mathrm{Hb}: 9.5 \mathrm{gm} \%$, TLC : 6800/cu.mm, DLC : N48\% L34\% E18\%,Urine R/M : gross- pyuria + , albumin - +++, pus cellsplenty/hpf, Urine culture : No growth, negative for AFB.RBS :115 mgs\%, HIV status negative, XRay Chest showed a nodular, oval well circumscribed opacity in left upper zone? Hamartoma? tubercular lesion.

On examination, patient was thin built \& ill nourished, PR : 76/m , BP: 100/70 mm/hg. P/A: Soft, no tenderness, no organomegaly. On vaginal examination, cervix was normal, uterus atrophic, fornices free, no mass palpable, no tenderness.

Patient was posted for diagnostic laparoscopy. Intraoperative findings: large bowel loops, omentum, peritoneal surface studded with tubercles? Miliary abdominal tuberculosis. No evidence of ascites. One horn of uterus visualised, uterus was atrophic and adherent to anterior abdominal wall. Parietal peritoneum \& omental biopsy was collected.

Subsequently omental and peritoneal biopsy report was suggestive of Tuberculosis. Patient was started on anti Koch's treatment (AKT) and discharged. She is on regular follow up. There is an improvement in the physical well being of the patient, though her fertility potential is yet to be evaluated after completion of AKT.

DISCUSSION: Genital tuberculosis continues to be a major cause for female infertility in developing countries including India. Inspite of recent advances in medicine and availability of sophisticated investigative modalities, definitive diagnosis of GTB is elusive in absence of high clinical suspicion.

The most common initial symptom of genital tuberculosis is infertility with a reported incidence of $40-80 \%$.Chronic lower abdominal pain or pelvic pain is the second most common symptom seen in $20-50 \%$ of patients. Menstrual pattern may be disturbed in $10-60 \%$ of patients. The change may be towards menorrhagia or metrorrhagia as in other pelvic infections but oligomenorrhea or amenorrhea is more common. Abnormal vaginal discharge is not significant in endometrial TB. ${ }^{5}$

Haematological examination shows tendency towards lymphocytosis. Chest X-ray may show a picture of active or healed lesion. Microscopic hematuria, abacteriuric pyuria may suggest urinary tract involvement. Mantoux has limited utility in diagnosing active genital TB during child bearing age. However, in infertile women with a positive Mantoux test, laparoscopy should be advocated early. Endometrial biopsy (EB) for TB remains the most commonly used procedure. It detects $50-60 \%$ of patients with genital TB. A negative EB does not rule out pelvic involvement ${ }^{5}$. Due to the pauci bacillary nature of GTB, diagnosis by 
mycobacterial culture and histopathological examination (HPE) have limitations and low detection rate ${ }^{6}$. HSG is unreliable as a diagnostic tool \& can cause exacerbation of the disease. Laparoscopy is now a well recognized procedure in the diagnostic work up of patients with infertility. In a clinically suspicious case, EB should be followed by laparoscopy ${ }^{5}$

In our case, the diagnosis of bicornuate uterus had possibly misled the investigation, such that it had been focused upon as the main cause for her infertility and thereby the real diagnosis of tuberculosis was neglected. Women with bicornuate uterus can expect a reasonable success in delivering a living child (60\%).There is no evidence of primary infertility in women with bicornuate uterus. ${ }^{7}$

The treatment of GTB should begin with anti tuberculous therapy. Historically pregnancy outcomes after treatment have been generally poor and the risks of ectopic pregnancy and spontaneous abortion are increased. Pregnancy rates can now be significantly improved with In vitro fertilization (IVF) $(16 \%$ - $25 \%$ per transfer) but success remains dependent on normal endometrial function. Success rate is high with trophic endometrium and reduces to nil in cases of atrophic endometrium. An initial investigation of endometrium given the high rate of uterine extension is warranted prior to initiating IVF in patients with GTB ${ }^{8}$.

TB PCR test, considered as a gold standard for assessment of endoscopic features, is not $100 \%$ sensitive and specific. Like any other bacteriological test available for diagnosing TB, it is known to give a small number of false-positive and -negative results.

For the endoscopic features which are considered suggestive of TB, there are no defined criteria in literature. However, in a study conducted by Sharma JB et al, the findings on laparoscopy suggestive of tuberculosis were tubercles on peritoneum $(12.9 \%)$ or ovary $(1.2 \%)$, tubo-ovarian masses $(7.1 \%)$, caseous nodules (5.8\%), encysted ascitis in $7.1 \%$ women. Various grades of pelvic adhesions were seen in $65.8 \%$ of women. The various findings on fallopian tubes were normal looking tubes in $7.1 \%$, inability to visualize in $14.1 \%$, presence of tubercles on tubes in $3.52 \%$, caseous granuloma in $3.52 \%$, hydrosalpinx in $17.6 \%$, pyosalphinx, beaded tube with tobacco pouch appearance in $2.35 \%$ women. One of the two tubes was patent in $23.3 \%$ cases, while they were either not seen or blocked at various sites with cornual end being most common ${ }^{10}$.

This case report could prove to be an eye opener for gynaecology practitioners dealing with infertility patients in our country. Tuberculosis, in the past was considered as a leading diagnosis in cases of unexplained infertility in developing countries. In the present era, with the introduction of DOTS, we seem to be coming across fewer patients with GTB as a cause for infertility. However, even in the present scenario, in our country, there may be many such cases of GTB wandering from pillar to post for want of appropriate diagnosis and treatment.

CONCLUSION: We have made an attempt to report this case to re-emphasize the existence and high incidence of genital TB in our country. This may just be the tip of the iceberg. The Gynaec infertility specialists should keep tuberculosis in mind while evaluating infertility. The diagnosis of genital TB remains an enigma. Early diagnostic laparoscopy may be the only efficient tool to aid the evaluation.

CONSENT: Consent for publication of this case report was taken from the patient, copy of which is available with the corresponding author. 
ACKNOWLEDGEMENT: We would like to extend our heartfelt gratitude to the heads of Dept, Obstetrics \& Gynecology and Pathology.

\section{REFERENCES:}

1. Muir DG, Belsey MA. Pelvic inflammatory disease and its consequences in the developing world. Am J Obstet Gynecol 1980;138:913-28.

2. Schaefer G. Female genital tuberculosis. Clin Obstet Gynecol. 1976;19:223-39

3. 3.Ben Youssef LB, Chelli H, Belhadj A. Current anatomo-clinical aspects of genital tuberculosis in women. Apropos of 49 cases. J Gynecol Obstet Biol Reprod (Paris) 1985;14:59-65.

4. Varma TR. Genital tuberculosis and subsequent fertility. Int J Gynecol Obstet. 1991;35:111.

5. Gowri Gandhi, Sumita Mehta, Swaraj Batra. Genital Tuberculosis. Infections in Obstetrics \& Gynaecology. $1^{\text {st }}$ ed. Jaypee Brothers Medical Publishers; New Delhi ; 2006 : 77-92.

6. R.B.P. Thangappah, C.N. Paramasivan, Sujatha Narayanan. Evaluating PCR, culture \& histopathology in the diagnosis of female genital tuberculosis. Indian J Med Res. 2011 July; 134(1): 40-46.

7. Schorge, Schaffer, Halvorson, Hoffman, Bradshaw, Cunningham. Anatomic Disorders. Williams Gynecology. $1^{\text {st }}$ ed. The Mc Graw - Hill Companies; New Delhi; 2008 : 402-425.

8. Marcelle I. Cedars. Common medical conditions and their impact on reproductive functions. Infertility - Practical pathways in obstetrics and gynecology. $1^{\text {st }}$ ed. The Mc Graw - Hill Companies; USA ; 2005 : 91-145.

9. Baxi Asha ,Neema Hansal, Kaushal Manila, Sahu Priti ,Baxi Dhawal. Genital Tuberculosis in Infertile Women: Assessment of Endometrial TB PCR Results with Laparoscopic and Hysteroscopic Features. J Obstet Gynaecol India. 2011 June; 61(3): 301-306.

10. Sharma JB, Roy KK, Pushparaj M, Kumar S, Malhotra N, Mittal S. Laparoscopic findings in female genital tuberculosis. Arch Gynecol Obstet. 2008 Oct;278(4):359-64.

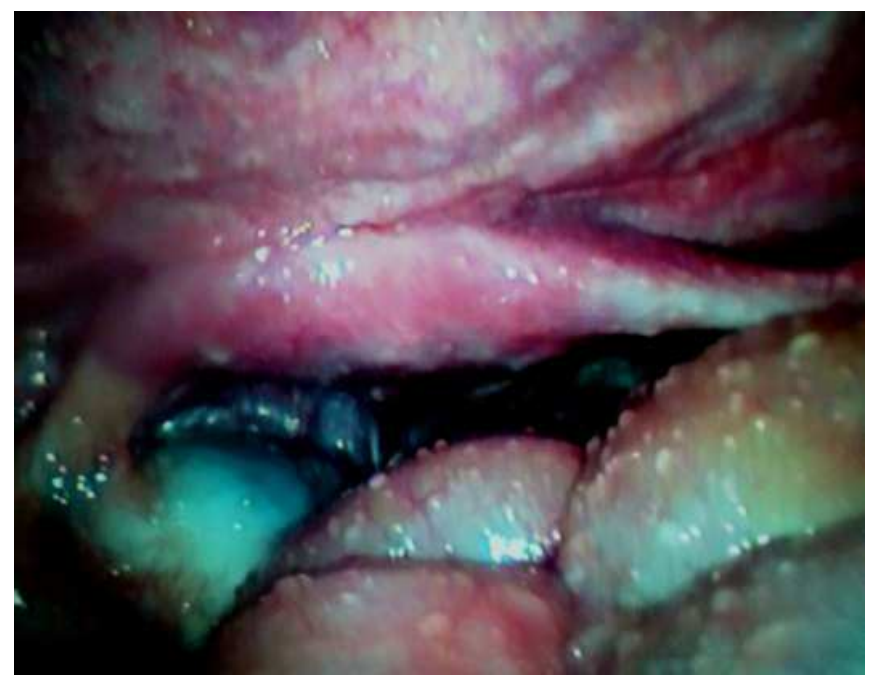

Figure I : Laparoscopy view showing uterus, ovary studded with tubercles 


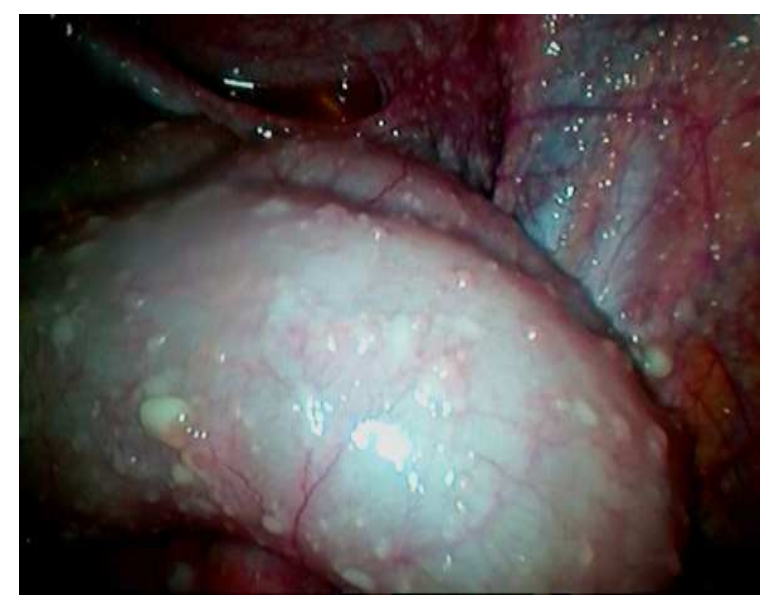

Figure II : Laparoscopy view showing bowel and parietal peritoneum with tubercles

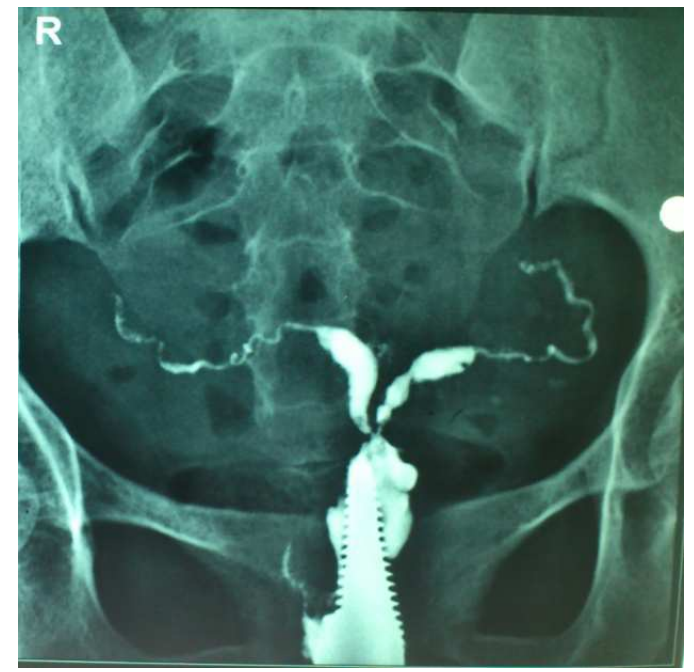

Figure III HSG picture showing bicornuate uterus, beaded appearance of both fallopian tubes seen along the entire length, no free spill.

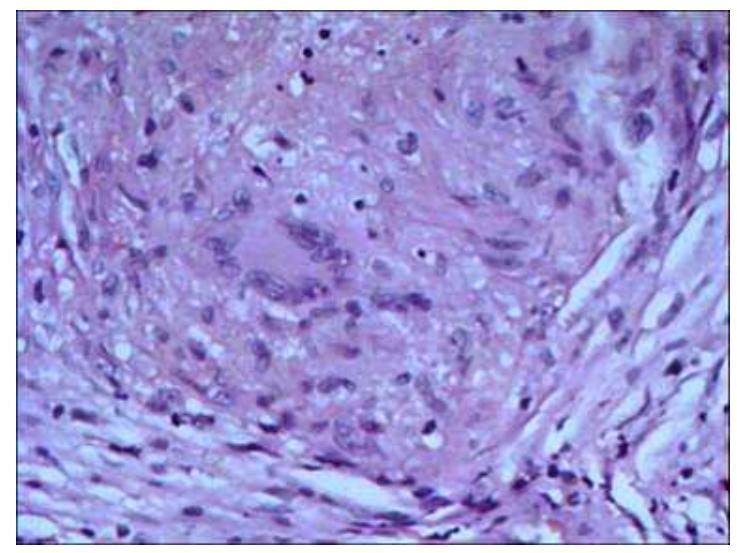

Figure IV: Histopathology picture (40X) showing giant cell with granuloma formation suggestive of tuberculosis. 